\title{
Comparative Analysis and Implementation of Efficient Digital Image Watermarking Schemes
}

\author{
M. Ali Qureshi, Abdul Aziz, Bilal Ahmed, Ayesha Khalid, and Humera Munir
}

\begin{abstract}
Due to advancement in digitized media and enormous growth in multimedia systems, there was a need of an efficient and robust watermarking technique for digital images that can be used for ownership copyright of multimedia objects, trademarks, finger printing authentication etc. Digital Image watermarking can be done both in spatial domain and frequency domain. In this paper we have implemented watermarking algorithm in frequency domain, i.e. by using Discrete Cosine Transform (DCT) and Forward Hadamard Transform (FHT). For that purpose, a watermark image of size $64 \times 64$ is embedded in original image of size $512 \times 512$ using both methods. The resultant watermarked image is experienced under different types of attacks. Original watermark is recovered by inverse DCT-based and FHT-based watermarking. The visual performance and robustness of both watermarking algorithms is measured in terms of Normalized Cross Correlation (NCC) and Peak Signal to Noise Ratio (PSNR). At the end, comparative analysis of both watermarking techniques is made.
\end{abstract}

Index Terms-Watermarking, DCT, FHT, PSNR, NCC, impercptibility, roboustness.

\section{INTRODUCTION}

Digital watermarking is a technology of inserting a pattern of bits into digital image for the copyright protection. Bits representing the watermark must be scattered throughout the image in such a way that it cannot be identified and manipulated .Watermark must fulfill two important properties. Firstly it must be robust and secondly it must be imperceptible to the attacks introduced. Robustness means how much strong the watermark is. The stronger it is, greater will be the chance for it to resist transformations. Whereas imperceptibility refers to the visual quality of the watermarked image. A digital watermark must be robust and imperceptible enough to survive changes, it is embedded in.

In the recent years a lot of research is done to evaluate the techniques of digital watermarking and they are divided into two main groups [1]. First is the Spatial domain approach and the other is Frequency domain approach [2]. Spatial domain is the simplest technique to embed a watermark into the least significant bit (LSB) of the image pixel. The spatial domain methods are simpler than frequency domain methods. But the watermark can easily be erased by lossy image compression. Where as in the frequency domain operations are performed on the Fourier transform of the image, rather than the image itself. The transforms included in frequency domain are

Manuscript received April 9, 2012; revised July 26, 2012.

M. A. and A. Aziz are with Islamia University of Bahawalpur, Pakistan (e-mail: ali.qureshi@iub.edu.pk; abdul.aziz@ iub.edu.pk). mainly DCT, DFT, DWT and FWHT [3].

There are many schemes which have been introduced to improve the watermark but some of them are used along with the others, such as Slant, Hartley and Hadamard. Hadamard is the most popular one. Bhatnagar and Raman [4] proposed a robust watermark scheme with multi resolution WHT, using singular value decomposition (SVD). Li, Wang, Song and Wen [5] proposed a blind multiple watermark schemes using Hadamard transform. It was presented that the scheme is invisible and robust against attacks. In this paper we are implementing watermarking algorithms using DCT and FHT. The performance and robustness of both watermarking algorithms are compared against several image attacks.

\section{A. Discrete Cosine Transform (DCT)}

Discrete cosine transform (DCT) is used to express a finite data points sequences in terms of a sum of cosine functions oscillating at different frequencies [6]. DCT has many applications in the field of engineering and science. Some common applications are lossy compression of images and audio (in which very small high frequency components are discarded) and spectral methods used for numerical solution of partial differential equations. In fact DCT is similar to discrete Fourier transform (DFT). But it differs from DFT because calculations in DCT involve real numbers only.

Discrete Cosine Transform (DCT) de-correlates the image data like other transforms. After de-correlation each transform coefficient can be encoded independently without losing compression efficiency.

In the DCT based watermarking scheme, the watermark bits are embedded in each $\mathrm{N} * \mathrm{~N}-\mathrm{DC}$ block of the host image [7]. The IDCT is used to reconstruct the watermark in which after the watermark is embedded into the host image. DCT is suitable for watermarking when the channel noise is low, but low channel noise is not always the reason for it. The formula for calculating 1-D DCT is given below.

$$
F(u)=\left(\frac{2}{N}\right)^{\frac{1}{2}} \sum_{i=0}^{N-1} A(i) \operatorname{Cos}\left[\frac{\Pi}{2} \frac{u}{N}(2 i+1)\right] f(i)
$$

where the 2D-DCT can be calculated as:

$$
\begin{aligned}
& \left.F(u, v)=\left(\frac{2}{N}\right)^{\frac{1}{2}}\left(\frac{2}{M}\right)\right)^{\frac{1}{2}} \sum_{i=0}^{N-1} \sum_{j=0}^{M-1} A(i) A(j) \operatorname{Cos}\left[\frac{\Pi}{2} \frac{u}{N}(2 i+1)\right] \\
& \operatorname{Cos}\left[\frac{\Pi}{2} \frac{v}{N}(2 j+1)\right] f(i, j)
\end{aligned}
$$

\section{B. 2D- Fast Hadamard Transform (FHT).}

The 2-Dimentional Hadamard transform is mainly used for 
image compression and image processing operations. In this paper, we are dealing with image data and Hadamard transform is used only for watermark embedding and extraction of watermark. If we represent original image by $[\mathrm{U}]$ and the transformed image by [V] then 2D Hadamard transform is evaluated as [6].

$$
[V]=H_{n}[U] H_{n} / N
$$

where

$H_{n=} N x N$ Hadamard matrix for $\mathrm{n}=0,1,2,3 \ldots \ldots$

In this scheme the gray scale image is embedded as the watermark. For embedding process, the watermark image is decomposed into Hadamard coefficients. There are many advantages of Hadamard transform like that elements of the transform matrix $\mathrm{H}_{\mathrm{n}}$ are simple i.e. they are in the form of 1 or -1 . In addition to that rows and columns of Hadamard matrix are orthogonal. The Hadamard transform matrix also exhibit following property.

$$
\mathrm{H}_{\mathrm{n}}=\mathrm{H}_{\mathrm{n}}^{*}=\mathrm{H}^{\mathrm{T}}=\mathrm{H}^{-1}
$$

where $H_{n}$ has $\mathrm{N}$ orthogonal rows and $H_{n} H_{n}=N I$ where $\mathrm{I}$ is the Identity matrix and $H_{n} H_{n}=N_{n} H_{n}^{-1}$

To make the watermark completely hidden, a visual model based on the factors of original image, like edges and texture are important to determine the strength factor of watermark. This strength factor is scaled with the watermark coefficients to the similar range to the coefficients from the Hadamard coefficients of the sub-block of the host image. For hiding the watermark, Middle and High Frequency bands are suitable in Hadamard transform as compared to the other high coding gain transforms like DCT at high noise conditions [7]. FHT algorithm is found to be the most computationally efficient that provides the necessary conditions like robustness and good image quality which is also known as property of imperceptibility. Due to shorter processing time and ease of hardware implementation, FHT is more advantageous than other orthogonal transformation techniques like DCT and DWT.

\section{Fast Walsh Hadamard Transform (FWHT}

Hadamard transform matrix is the orthogonal square matrix which only consists of 1 and -1 as the element value. This transform is also known as Walsh-Hadamard transform. $\mathrm{H}_{1}$ is the smallest Hadamard matrix, and it is defined as:

$$
\begin{gathered}
H_{N}=\frac{1}{\sqrt{2}}\left(\begin{array}{ccc}
H_{N-1} & H_{N-1} \\
H_{N-1} & -H_{N-1}
\end{array}\right) \\
H_{2}=\left[\begin{array}{cccc}
1 & 1 & 1 & 1 \\
1 & -1 & 1 & -1 \\
1 & 1 & 1 & 1 \\
1 & -1 & 1 & -1
\end{array}\right]
\end{gathered}
$$

where the number of sign changes along each row in the above matrix [ $\left[\begin{array}{llll}0 & 3 & 1 & 2\end{array}\right]$ respectively.

The number of the signs changes in each row of the matrix is called sequence of the row. These rows can be considered to be samples of rectangular waves with the sub period of $\mathrm{I} / \mathrm{N}$ units. These continuous functions are Walsh functions [8]. The orthogonal Hadamard matrix must satisfy the following condition.

$$
H H^{T}=I
$$

The $\mathrm{H}$ is the Hadamard matrix, $H^{T}$ is the inverse Hadamard matrix, and I is the unitary matrix. By using Fast Walsh Hadamard transform algorithm, $N \log N$ operations are required for the computation of Hadamard transform. Consider $\mathrm{y}$ is the signal vector, $\mathrm{Y}$ is the spectrum vector, and $\mathrm{H}$ is the Hadamard matrix. The Walsh-Hadamard transform (WHT) and Inverse Walsh Hadamard transform (IWHT) are defined as

$$
\begin{aligned}
W H T(y) & =Y=H_{y} \\
\operatorname{IWHT}(Y) & =y=H Y
\end{aligned}
$$

The WHT and IWHT are the forward and inverse of FWHT, respectively. The sequency ordered Walsh Hadamard transform $\left(\mathrm{WHT}_{\mathrm{w}}\right)$ can be obtained by first carrying out the first $\mathrm{WHT}_{\mathrm{w}}$ and then reordering the components of Y [9].

\section{The Proposed Digital Watermarking Schemes}

\section{A. Watermark Embedding}

- The original Image is chosen of size $512 \times 512$ and watermark image is chosen of size $64 \times 64$.

- The original image is converted into its gray scale components.

- Compute DCT and FHT of watermark image. For watermark of size $64 \times 64$ we have 2056 coefficients

- $\quad$ Divide the original image into blocks of size $8 \times 8$. For original image of size $512 \times 512$ we have 2056 $8 \times 8$ blocks.

- Compute DCT and FHT of each sub-block.

- Take one DCT or FHT middle frequency coefficient from original image sub-block and replace it with DCT or FHT coefficient of watermark image.

- Repeat the process for each block.

- Compute inverse DCT and inverse FHT and watermarked image is obtained as shown in Figure 1.

\section{B. Watermark Extraction}

- The watermarked image is divided into $8 \times 8$ blocks.

- The DCT and FHT is computed for each sub-block and the corresponding coefficient is extracted from position where it was placed while watermark embedding.

- These coefficients are stored in a vector of size $1 \times 2056$.

- Repeat the process for each block.

- In the end, the coefficients stored in a linear array are rearranged in 64x64 matrix.

- The watermark is recovered by taking the inverse DCT and inverse FHT of the above matrix as shown in Fig. 2. 


\section{Performance Measurements}

There are a numerous measures used for determining the effectiveness of watermarking algorithm and which directly or indirectly influence the properties of inserted watermark. In this paper we are using Peak Signal to Noise Ratio (PSNR) for determining imperceptibility of watermarked image and Normalized Cross Correlation (NCC) for determining the robustness of proposed watermarking algorithms against various attacks.

1) Peak signal to noise ratio (PSNR)

We use Peak Signal to Noise Ratio to measure the visual fidelity or imperceptibility between the original image and the watermarked image [10]. PSNR can be calculated by using the given formula.

$$
\operatorname{PSNR}(d B)=10 \log _{10}\left[\frac{R^{2}}{\sum_{u=1}^{M} \sum_{v=1}^{N}[X(u, v)-\bar{X}(u, v)]^{2}}\right]
$$

For $\mathrm{u}=1,2,3 \ldots \mathrm{M}$ and $\mathrm{v}=1,2,3, \ldots \ldots \mathrm{N}$

$\mathrm{X}=$ Original image.

$\mathrm{X}^{\prime}=$ Watermarked image

$\mathrm{R}=$ Maximum variation in input image data type

$\mathrm{N}$ and $\mathrm{M}$ are dimensions of input image as columns and rows respectively.

2) Normalized cross correlation (NCC)

The NCC is used to indicate the similarity between two images. In our case, it is used to determine how similar the extracted watermark with that of the original watermark image is. The value of NCC defines the strength of robustness of extracted watermark [10]. It can be calculated by the following formula.

$$
N C C=\left[\frac{\sum_{u=1}^{M} \sum_{v=1}^{N}[W(u, v) \bar{W}(u, v)]}{\sum_{u=1}^{M} \sum_{v=1}^{N}[W(u, v)]^{2}}\right]
$$

$W=$ original watermark

$W^{\prime}=$ Extracted watermark.

$M$ and $\mathrm{n}$ are rows and columns of watermark image respectively

\section{EXPERIMENTAL RESULTS}

The performance and robustness of watermarking algorithms based on DCT and FHT is measured in terms of PSNR and NCC. For that purpose different types of attacks have been implemented on the watermarked image and watermark is recovered using inverse watermarking algorithms based on DCT and FHT. Following attacks have been added to the watermarked image.

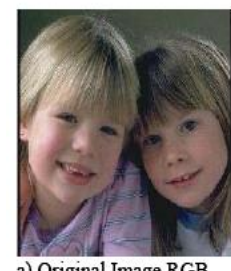

a) Original Image RGB

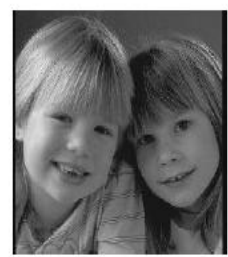

b) Original Image Gray

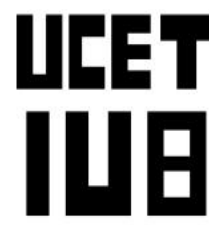

c) Watermark
Fig. 1. Original image and watermark image
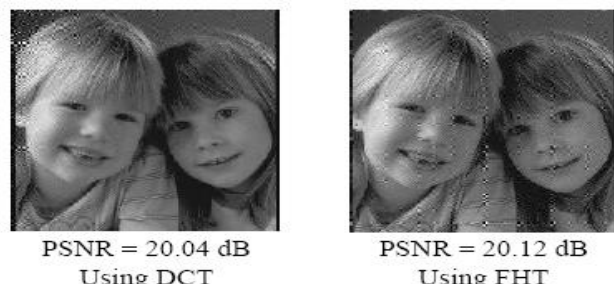

Fig. 2. Watermarked image using DCT and FHT

A. Lowpass Filtering

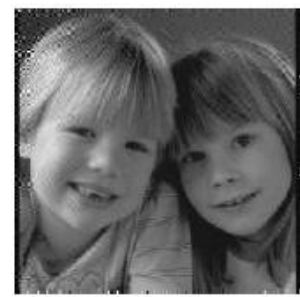

PSNR $=22.59 \mathrm{~dB}$

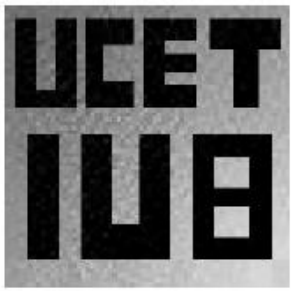

$\mathrm{NCC}=0.6257$
Fig. 3. DCT based watermarked image after lowpass filtering and recovered watermark

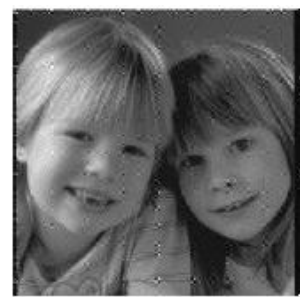

PSNR $=23.075 \mathrm{~dB}$

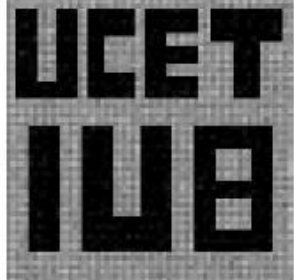

$\mathrm{NCC}=0.559$
Fig.4. FHT based watermarked image after lowpass filtering and recovered watermark

\section{B. Median Filtering}

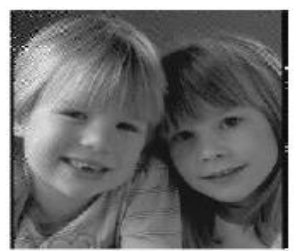

PSNR $=23.148 \mathrm{~dB}$

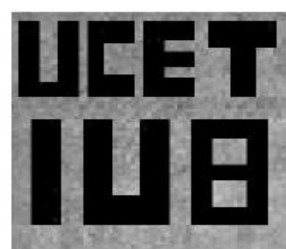

$\mathrm{NCC}=0.579$
Fig. 5. DCT based watermarked image after median filtering and recovered watermark

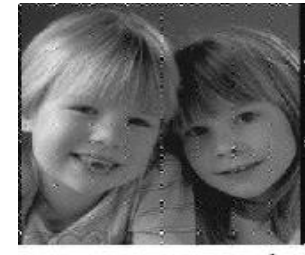

PSNR $=20.81 \mathrm{~dB}$

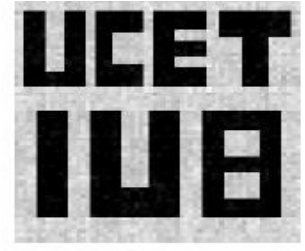

$\mathrm{NCC}=0.815$
Fig. 6. FHT based watermarked image after median filtering and recovered watermark 


\section{Highpass Filtering}

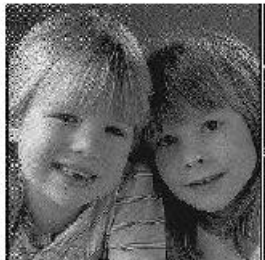

$\operatorname{PSNR}=12.02 \mathrm{~dB}$

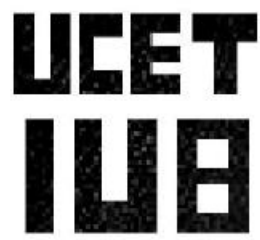

$\mathrm{NCC}=0.99$

Fig. 7. DCT based watermarked image after highpass filtering and recovered watermark

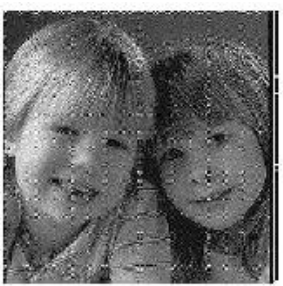

PSNR $=11.156 \mathrm{~dB}$

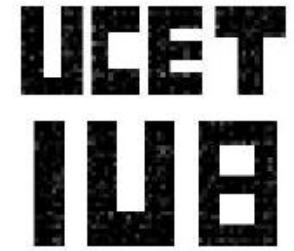

$\mathrm{NCC}=0.99$

Fig. 8. FHT based watermarked image after highpass filtering and recovered watermark

\section{Addition of Gaussian Noise}

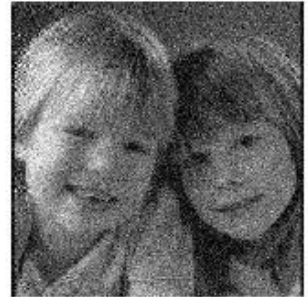

PSNR $=18.645 \mathrm{~dB}$

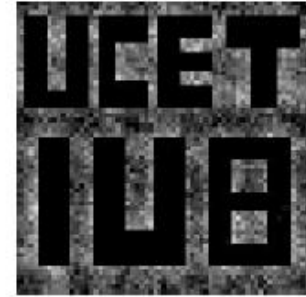

$\mathrm{NCC}=0.368$

Fig. 9. DCT based watermarked image after addition of Gaussian noise and recovered watermark

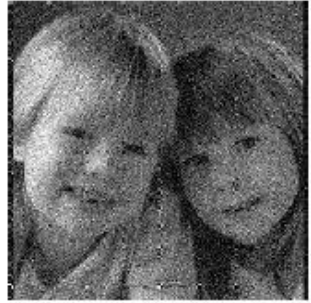

PSNR $=18.482 \mathrm{~dB}$

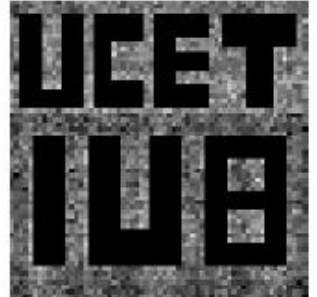

$\mathrm{NCC}=0.436$

Fig. 10. FHT based watermarked image after addition of Gaussian noise and recovered watermark

\section{E. Addition of Salt \& Pepper Noise}

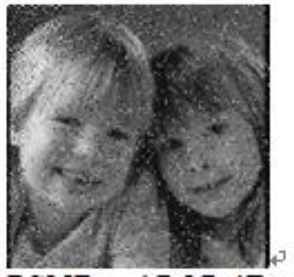

$\mathrm{PSNR}=17.37 \mathrm{~dB}$

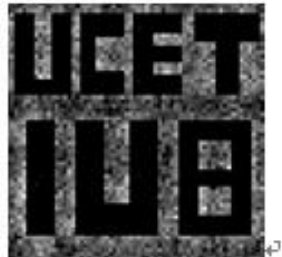

$\mathrm{NCC}=0.369$

Fig. 11. DCT based watermarked image after addition of salt $\&$ pepper noise and recovered watermark

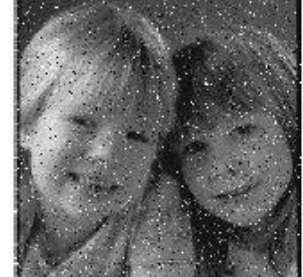

PSNR $=17.206 \mathrm{~dB}$

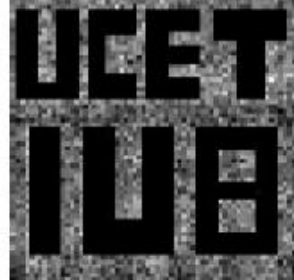

$\mathrm{NCC}=0.430$
Fig. 12. FHT based watermarked image after addition of salt $\&$ pepper noise and recovered watermark

\section{CONCLUSION}

In this paper, efficient image watermarking schemes are proposed by using DCT and FHT. Experimental results shows that both schemes are very efficient and robust against various non-geometric attacks.. But FHT technique is much batter than DCT because in FHT technique imperceptibility is very good. The recovered watermark is also resembles with the original watermark and it can be seen easily from the test results that NCC values is greater in case of FHT as compared to DCT. The computation time for watermarking algorithm using FHT is also very low as compared to DCT.

\section{ACKNOWLEDGMENT}

We are thankful to our parents and our teachers who guided us through out our career path. We are also thankful to all our dearest friends who provided us encouragement to complete the tasks. We are indebted to our Principal, Prof. J.M.Keerio for his sincere efforts in providing research environment.

\section{REFERENCES}

[1] R. G. V. Schyndel, A. Z. Tirkel, and C. F. Osborne, "A digital watermark," in proc. IEEE int. conf. image processing, vol. 2, pp 86-90.1994

[2] J. J. Cox, J. Kilian, F. T. Leighton, and T. Shamoon, "Secure Spread spectrum watermarking for multimedia," IEEE trans. image processing, vol. 6, pp.1673-1688, Dec.1997

[3] A. Marjuni, R. Logeswaren, M. F. A. Fauzi, "An image watermarking scheme based on FWHT-DCT," IEEE int. conf. networking and information technology, 2010.

[4] G. Bhatnagar and B. Raman, "Robust watermarking in multi resolution Walsh-Hadamard Transform," in Proc. IEEE international advance computing conference (IACC 2009), India, March 2009, pp. 894-899.

[5] H. Li, S. Wang, W. Song, and Q. Wan, "Multiple watermarking using Hadamard transform," Lecture notes in computer science, 2005 pp.767-772

[6] K. Rao and P. Yip, "Discrete Cosine Transform: algorithms," advantages. Applications. Academic Press, USA, 1990.

[7] F. A. P. Petitcolas, R. J. Anderson and M. G. Kuhn, "Information Hiding- A survey," in Proceedings of the IEEE, Special Issue on Protection of Multimedia Contents, vol. 87, no. 7, July 1999, pp. 1062 -1078 .

[8] T. S. Anthony, H. J. Shen, K. K. A. Chow, and J. Woon, "Robus Digital image-in -image watermarking algorithm using the fast Hadamard Transform," IEEE international conference, 2003.

[9] A. N. Akansu and R. A. Haddad, "multi resolution signal decomposition transforms,subbands and wavelets,academic press inc,"'1992

[10] W. K. Pratt, "Digital image processing, fourth edition," wiley-interscience, John wiley and sons, inc, California, 2007. 